\title{
Author Index Vol. 33, 1996
}

Alexander, G.E. 127 Atobe, M. 132 Avanzini, P. 173

Barak,YY. 164 Bieck, P.R. 160 Blatný, M. 138 Brambilla, F. 173 Bucci, P. 48 Buchsbaum, M.S. 1 Bunney, W.E., Jr. 1 Burgin, R. 28

Caccavari, R. 173 Cardinali, D.P. 147 Chang,W.-H. 12 Chiang, T.-S. 12 Chien, C.-P. 12 Chiodera, P. 71 Chwatal, K. 80 Coiro, V. 71 Coppola, R. 32

Delsignore, R. 173 Demmer, M. 164 Di Gennaro, C. 71 Dirlich, G. 90 Dolev, E. 169 Drago, F. 186 Drake, M.E., Jr. 97

Elizur, A. 164

Fertomani, G. 173 Freedman, R. 113 Fukuda, H. 66

Galderisi, S. 48 Gargiulo, P.A. 189 Gavish, M. 28 Gerra, G. 173 Gleiter, C.H. 160 Goldman, R.G. 127 Graeff, F.G. 189 Gräsbeck, A. 118 Grözinger, M. 155 Guinjoan, S.M. 147 
Hagnell, O. 118 Haier, R.J. 1 Hall, S.R. 202 Harel, M. 164 Harr, A. 210 Hartmann, A. 90 Havelec, L. 80 Hayakawa, H. 17 Hayashi, T. 55 Herrmann, W.M. 32,160 Heuser-Link, M. 90 Hiramoto, K. 182 Holsboer, F. 90 Hosokawa, K. 76 Hrdlicka, M. 138 Hu, W.-H. 12

Ichikawa, Y. 76 Isberg, P.-E. 118 Ito, M. 182 Iwahashi, K. 76

Jann, M.W. 12 Jobert, M. 32,160

Kagaya, A. 55 Kariya, T. 132 Katz, M. 1 Kimhi, R. 164 Klieser, E. 196 Knott, VlJ. 210 Komura, S. 85 König, P. 80 Koopman, P.A.R. 100 Kozaki, N. 85 Krauth, J. 196 Krijzer, F.N.C. 100 Krumrey, K. 90 Kubota, M. 132

Lehmann, E. 196 LePira, F. 186 Levine, J. 169 Levy, D. 164 Lin, H.-N. 12 Lococco, J. 147 Lombardo, R.J. 106 
Maestri, D. 173 Maj, M. 48 Malaguarnera, M. 186 Mann, K. 41 Mashiah, M. 169 Mignone, M.L. 48 Miura, T. 21 Miyatake, R. 76 Monica, C. 173 Moráñ, M. 138 Motohashi, N. 17,55,60 Mucci, A. 48 Mukherjee, S. 127 Muraoka, M. 17 Murer, M.G. 106

Nakagawara, M. 132 Nakamura, K. 76 Newell, S.A. 97 Nicoletti, G. 186 Nishida, A. 60,66

Ogata, M. 85 Ohnishi, T. 76 Okada, T. 182 Okamoto, Y. 17,55

Pakalnis, A. 97 Pantev, M. 32 Prohovnik, I. 127

Raffaele, R. 186 Rampello, L. 186 Rapoport, A. 169 Rastelli, G. 71 Reali, N. 173 Reimann, I.W. 160 Riedl, F. 80 Riquelme, L.A. 106 Rorsman, B. 118 Röschke, J. 41,155 Rozinková, J. 138 Rutledge, J.H., III 113

Sackeim, H. 127 Saito, H. 60,66 Schubert, H. 80 
Schultes, H. 80 Schulz, H. 32 Schulze Mönking, H. 142 Shimizu, M. 60,66 Siegel,|B.V., Jr. 1 Siri, L.N. 147 Smith,|A.P. 202 Souza Silva, M.A. de 189 Strik, W.K. 168 Suwaki, H. 76

Tomaz, C. 189 Turri, M. 160

Uemura, K. 85

Vachutka, J. 138 Vescovi, P.P. 71 Viana, M.B. 189 Vogl, L. 90 Volpi, R. 71

Waldo, M. 113 Weizman, R. 28 Wesselmann, U. 142 Windgassen, K. 142 Wouters, P.A.W.M. 100 Wu, J. 1 Wurthmann, C. 196

Yamaji, T. 55 Yamawaki, S. 17,55,60,66 Yannielli, P.C. 147 Yayama, K. 85 Yoneyama, H. 76 Yoshida, T. 85 Yoshimoto, K. 85 Young,D.A. 113 Yui, K. 21 Zaimovic, A. 173 Zanuto, B.S. 106 Zemishlany, Z. 127 